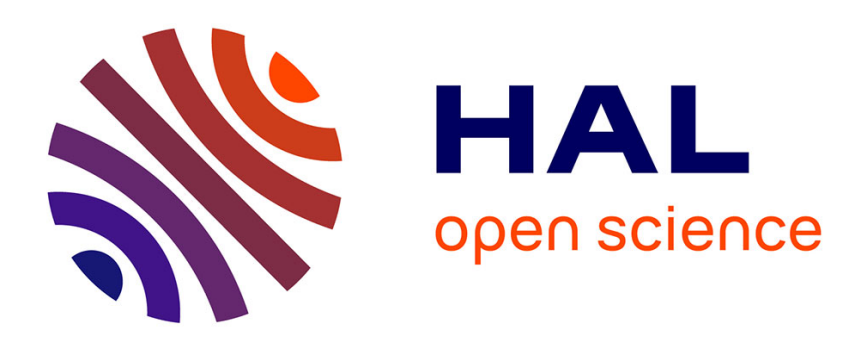

\title{
New Understanding of the Wave-Particle Dualism and its Application to Ferrites
}

\author{
S. Iida
}

\section{To cite this version:}

S. Iida. New Understanding of the Wave-Particle Dualism and its Application to Ferrites. Journal de Physique IV Proceedings, 1997, 07 (C1), pp.C1-173-C1-174. 10.1051/.jp4:1997162 . jpa-00255109

\section{HAL Id: jpa-00255109 https://hal.science/jpa-00255109}

Submitted on 1 Jan 1997

HAL is a multi-disciplinary open access archive for the deposit and dissemination of scientific research documents, whether they are published or not. The documents may come from teaching and research institutions in France or abroad, or from public or private research centers.
L'archive ouverte pluridisciplinaire HAL, est destinée au dépôt et à la diffusion de documents scientifiques de niveau recherche, publiés ou non, émanant des établissements d'enseignement et de recherche français ou étrangers, des laboratoires publics ou privés. 


\section{New Understanding of the Wave-Particle Dualism and its Application to Ferrites}

S. Iida

University of Tokyo, 4-32-11 Funabashi, Setagaya-Ku, Tokyo 156, Japan

Abstract. The solution of the wave-particle duality problem and the EPR paradox has been obtained by the unified frame in physics. This paper describes briefly its outline for the benefit of the scientists and engineers in ferrites.

In 1974, the author found ccc-electron(circular current c-number; c: classical) [1] and, in 1993, the unified frame in physics has been established [2-4]. Since both the classical and quantal physics have been unified, the two languages and concepts can be used simultaneously to solve difficult problems. Here the application to the EPR(Einstein-Podolsky-Rosen) paradox and the wave-particle duality problem [5] will be introduced [6]. In this unknown area of physics, it happens that there is no adequate physical concept nor language, so that, every existing or newly found concept should be used boldly, if it is most close to the subject, at least until the better concept has been found. We call this the frontier notion principle. It is noted that, in this most subtle problem, physical and observational uncertainties have mixed together to make everything indefinite.

The photon is known to have zero-point modes with the energy of $h v / 2$. Accordingly, the photon mode is present even without a photon. A superconductor microwave cavity has the $Q$ value of $3 \times 10^{10}$ at $0.5 \mathrm{~K}$ [7], and the rate of a spontaneous emission in the tuned frequency from the excited atom in the cavity is enhanced by the same factor [8]. Then, we shall use the concept and language that the amplitude of the zero-point photon mode at this frequency is enlarged by the factor of $Q$ [9]. "Null existence" is defined for this. These concepts and language are a typical application of the frontier notion principle. Supported by various dampingless phenomena of the superconductivity, the microscopic quantum world is regarded as an idealistic world where physical description is possible without using the terms of loss and damping.

Time reversal is accepted to electromagnetic waves. Proofs are given that the same is true for wave functions not only for the real Hamiltonian, but, also, in general, including the case of Stern-Gerlach experiments, where the two beams with opposite spin directions having splitted by the inhomogeneous magnetic fields, can reverse its time direction, to return to the single beam with the original spin direction. Therefore, the concept of retarded and advanced electromagnetic modes is extended into the time dependent wave functions in general. The EPR problems have been solved by requiring that the detectors must be included in the Hamiltonian or the Lagrangian of the system, and, the detection should be described by the advanced mode wave function. The time evolved wave function is replaced by the new concept of a complete mode, starting by a retarded mode, traveling, and, ending by an advanced mode. Besides the case of the delayed choice experiments, the particles having started the source know already how to settle finally, because they have chosen a certain complete mode. Whether the Maxwell equations or the Schrödinger or Dirac equations have such complete modes as their solutions is another problem, which is worthwhile to analyze, but, even if they are absent, it is regarded as due to the imperfectness of our mathematical framework, and, not due to the character of nature.

We have concluded that the microscopic world is a very active world with full of dynamic oscillations [10]. In addition to the well known zero-point photon oscillations, every particle(elementary as well as composite) in its rest frame, ${ }^{\circ} \mathrm{K}$, has de Broglie phasic oscillation, the frequency of which is exactly equal to its total energy over the Planck constant $\left(1.2356 \times 10^{20} \mathrm{~Hz}\right.$, $3.272 \times 10^{23} \mathrm{~Hz}$, and $4.5 \times 10^{24} \mathrm{~Hz}$ for electron, neutron, and ${ }^{20} \mathrm{Ne}$, respectively). As, relativistically, the time axis of ${ }^{\circ} \mathrm{K}$ has a slight component to a spacial axis of the laboratory frame, $\mathrm{K}$, the de Broglie oscillation in ${ }^{\circ} \mathrm{K}$ exhibits the wave aspect in $\mathrm{K}$. Therefore, the de Broglie wave packet of a moving particle is a relativistic side view of this very fast de Broglie phasic oscillation of the particle. We note that the electron wave functions of atoms have this aspect, and, de Broglie wave and wave function of the electron are essentially the same, although there are differences in the neglect and emphasis.

The total stationary wave function of a hydrogen atom in its rest frame ${ }^{\circ} \mathrm{K}$ is $\exp \left[-i\left(E_{\mathrm{p}}+E_{\mathrm{e}}+E_{\mathrm{SR}}\right) / h\right] \cdot \psi\left(r, r_{\mathrm{p}}\right)$. (1). Here, $E_{\mathrm{p}}=m_{\mathrm{p}} c^{2}$ and $E_{\mathrm{e}}=m_{\mathrm{e}} c^{2}$ are the rest energies of the proton and the electron, with the coordinates of $r_{\mathrm{p}}$ and $r$ Neglecting the kinetic energy of the proton, we have the Schrödinger Hamiltonian of $\mathrm{H}_{\mathrm{SR}}=p^{2} / 2 m_{\mathrm{e}}-e^{2} / 4 \pi r=E_{\mathrm{SR}}$ In (1), the rest energy de Broglie oscillation of the proton and the electron are usually neglected because they are uniform over the atom and have no effect for atomic state changes. The phase factor of (1) is the combined outcome of the physical truth and the complex number mathematical system, which we have to accept. Coexistence of the additivity of both the energies and the de Broglie frequencies of the system is beautifully represented, although no classical understanding to the additive nature of the de Broglie frequencies exists(an analogy may be the velocities in the Galilei transformation in Newtonian dynamics).

The c-number orbit of a s electron is a straight line, back and forth motion with the nucleus at the midpoint. Classical calculation gives easily for the frequency of the motion as a function of its Schrödinger energy as $v=\sqrt{8\left(-E_{\mathrm{SR}}\right)^{3} / m e^{4}}$. It is 
our finding that, by requiring the quantal condition $h v=\left|E_{\mathrm{SR}}\right|=-E_{\mathrm{SR}}$, the energy of the most simple s state i.e., $1 \mathrm{~s}$ state, $E_{\mathrm{SR}}=-m e^{4} / 32 \pi^{2} \hbar^{2}$, is deduced. Similar calculation for elliptic orbits gives the energy of $2 \mathrm{p}$ state, which is again the most simple angular momentum state. From these results we are obliged to conclude that the orbital motion of the q-number electron and the eigen wave function of the atom have a certain synchronization. Here the phasic oscillation of electromagnetic energy must be involved, because the energy includes the Coulomb term. We have made the order estimation of the oscillational frequency of the electric charge of ccc-electron(or ccc-muon as well) itself. Putting the radius and the circumference of the ring of ccc-electron as ${ }^{\circ} R$ and $2 \pi^{\circ} R$, we get $v_{\mathrm{e}} \sim c / 2 \pi^{\circ} R \approx m_{\mathrm{e}} c^{2} / 2 h$, which is almost identical to the de Broglie frequency of electron of $m_{\mathrm{e}} c^{2} / h$. We propose that the de Broglie frequency of electron might be equal to the internal structural frequency of the q-number electron itself, and, this principle might be effective to the other elementary particles.

In $1 \mathrm{~s}$ state of a hydrogen atom, the c-number radius calculated is $1.06 \AA$, being similar to the effective range of the square of the amplitude of $1 \mathrm{~s}$ wave function, of about $1 \AA$. Accordingly, in both the c-number and q-number structures of hydrogen atom, extensions of the location of the electron are similar. Applying the frontier notion principle, we postulate that the $q$-number extension of the electron itself is also in the same order of magnitude of that of the c-number electron, which is about $10^{-2} \AA$. This leads to a conclusion that, in general, the point charge electron should be replaced by the cce electron.

The extension of the de Broglie wave packet, however, can be quite large, such as a few to a hundred microns for electron, so that, by relativity, major arbitrary two portions of a de Broglie wave packet cannot communicate with each other, e.g., to readjust their phases. Whereas, because of the action through medium principle, the space-time must carry all the actions of particles by itself. Since de Broglie wave is quite general for all the particles, irrespectively of elementary and composite, we are obliged to conclude that it must be the existence somewhat independent from the mother particle, and, the reality which have the phasic oscillation must be the general space-time, or, the vacuum itself, and, electromagnetic oscillations are an important and indispensable component of the oscillation.

We have used a language in which the de Broglie oscillation and wave is regarded somewhat real. In this language, it is possible to say that strict agreement, or, matching of the four dimensional phases is most important for two de Broglie oscillations and waves to give the joint effects. When the matching is present, then, the superposed new de Broglie oscillation and wave has the new property, which is not equal to either of the two beams before joining. The q-number particle is located somewhere in either of the two beams, but, not in the both.

Here the submode concept will be introduced. The EPR problems have been solved by the complete mode concept in which each complete mode includes each detection procedure consisting of an advanced mode. Therefore, the current de Broglie wave function is regarded as consisting of millions of such concrete complete modes, or, submodes. Accordingly, there is no contradiction, if we assume that the wave function in $q$-number physics, in general, including those of the electrons in a stationary state of atoms, is composed of millions of such submodes, and, nature allows to include such submodes jointly in a single wave function, to make the result mathematically simpler, although it is possible to suppose that, in an instant, only one submode is active. It is noted that we have creation and annihilation operators also for the modes of the Fermion [11].

In conclusion, we have proposed that the c-number and $q$-number sizes of a particle are similar, but, the effect of the presence of the particle exists to the space-time, e.g., in the form of the de Broglie wave packet and the wave function, which is very large as compared to the assumed size of the particle. We may say that the mutual interaction or the intrinsic correlation between an elementary particle and the space-time, which includes zero-point photons, is strong and the resultant primary outcome is the wave function of that particle and/or the de Broglie oscillation and wave. This new view point will bring a new physical insight to the observed stability of atoms and molecules.

The complete mode concept will be applied to the ionic and electronic migration in ferrites. The ring diffusion will occur more frequently. The extra valence electrons in ferrites, such as on $\mathrm{Co}^{2+}$ against $\mathrm{Co}^{3+}$, and, on $\mathrm{Fe}^{2+}$ against $\mathrm{Fe}^{3+}$, may migrate more longer distance, and, this will explain why the relaxation times of electronic short range ordering in ferrites have much wider distribution than those of the ionic migration. It is noted that the recent studies on the higher valence transition metal oxides, the outer electrons of the higher valence ions, such as of $\mathrm{Co}^{4+}$, are more likely to have the band-like character, as compared to the lower valence ions, such as of $\mathrm{Co}^{3+}$. The formation of a band means that the migration distance becomes unlimited, which is not the case in the ionic migration.

The concept of the complete mode consisting of retarded and advanced modes has a neat application for the cold fusion to indicate the difficulty clearly, because, when the total energy of the system is not very high, the system cannot have such an advanced mode by which the two nuclei unite completely. This study will encourage the investigation for the room temperature superconductivity, although there must be many steps before arriving at the final goal.

\section{References}

[1] S. Iida, J. Phys. Soc. Jpn., 37, 1183 (1974).

[2] S. Iida, "Introduction to the Unified Frame in Physics". Original manuscript of this book was written in 1993 and copies were distributed in the world. The American Institute of Physics, the New York Academy of Sciences, Polish Academy of Sciences, Dr. V. Cagan of CNRS and Asahi News Paper Co. of Japan have a copy of the initial yersion of 1995.

[3] S. Iida, "Advances in Ceramics", Am. Cer. Soc., 15, 17-24 (1986).

[4] S. Iida, Proc. 6th Int. Conf. Ferrites, 1993, pp. 573-576.

[5] J. A. Wheeler and W. H. Zurek, Eds., "Quantum Theory and Measurement", 1983, Princeton Univ. Press, Princeton, N. J.

[6] Ref. [2], Chaps. XI, XII and XIII.

[7] F. Diedrich, J. Krause, G. Rempe, M. O. Scully and H. Walther, Physica, B 151, 247(1988).

[8] D. Kleppner, Phys. Rev. Lett. 47, 233 (1981).

[9] S. Iida, Discussion to H. Walther, Proc. 2nd. Int. Symp. Foundation of Quantum Mechanics, Tokyo, 1986, p. 35; to W.

Schleich, Proc. 3rd. Int. Symp. Foundation of Quantum Mechanics, Tokyo, 1989, p. 304.

[10] S. Iida, Proc. ISATQP-Shanxi'92, 1993, pp. 456-464. .

[1 l] J. J. Sakurai, "Advanced Quantum Mechanics", Addison-Wesley Pub. Co., Reading, Mass., et al., 1967, Chap. 2. 